\title{
Statistical Pattern Recognition Using the Normalized Complex Moment Components Vector
}

\author{
Stavros Paschalakis and Peter Lee \\ Electronic Engineering Laboratory, University of Kent at Canterbury, \\ Canterbury, Kent CT2 7NT, United Kingdom \\ sp11@ukc.ac.uk
}

\begin{abstract}
This paper presents a new feature vector for statistical pattern recognition based on the theory of moments, namely the Normalized Complex Moment Components (NCMC). The NCMC will be evaluated in the recognition of objects which share identical silhouettes using grayscale images and its performance will be compared with that of a commonly used moment based feature vector, the Hu moment invariants. The tolerance of the NCMC to random noise and the effect of using different orders of moments in its calculation will also be investigated.
\end{abstract}

\section{Introduction}

Geometric moments and moment based invariants have been widely used with success in the fields of image analysis and pattern recognition. A number of studies have been published by researchers which investigate their different properties, such as their applicability to different recognition problems ([1], [2], [3]), noise tolerance ([4], [5]), computational complexity and simplification ([6], [7]) and hardware implementation ([8], [9]).

This paper presents a new feature vector for statistical pattern recognition, the Normalized Complex Moment Components (NCMC), which is based on the theory of complex moments and maintains invariance with respect to translation, scale, illumination intensity and rotation transformations. The newly formed NCMC will be evaluated and compared with a commonly used moment based feature vector, the $\mathrm{Hu}$ Moment Invariants (HMI) ([10], [11], [12], [13]). The two feature vectors will be evaluated on a database of grayscale images which share identical silhouettes, relying on differences in their texture information for successful recognition. The effect of employing different classification measures in conjunction with the NCMC and HMI feature vectors will be investigated, as will the effect of employing moments of higher orders in the computation of both feature vectors. Finally, the noise tolerance of the NCMC will be compared against that of the HMI vector.

The rest of this paper is organized as follows: Section 2 will give a brief introduction to geometric moments and their normalization procedures. Section 3 will provide an analysis of the NCMC in detail, followed, in Section 4, by a brief description of the $\mathrm{Hu}$ moment invariants feature vector. Finally, the comparative results of this study and conclusions will be presented in Sections 5 and 6 respectively. 


\section{Geometric Moments}

For a discrete two-dimensional density distribution function $g(x, y)$ (e.g. a twodimensional image) with $x=0,1, \ldots, M$ and $y=0,1, \ldots, N$, the geometric moments $m_{p q}$ of order $p+q$ are defined as

$$
m_{p q}=\sum_{x=0}^{M} x_{y=0}^{N} x^{p} \cdot y^{q} \cdot g(x, y)
$$

Geometric moments are not directly suitable for object recognition since changes in the object's position, orientation, scale (i.e. distance from optical sensor) and the intensity of illumination will cause their values to change. A set of normalization procedures needs to be applied so that invariant properties may be established.

Translation invariance (i.e. invariance with respect to the object's position in the image scene) is achieved by calculating the central moments $\mu_{p q}$. These can be calculated directly from the ordinary moments using

$$
\mu_{p q}=\sum_{r=0}^{p}{ }_{s=0}^{q}\left(\begin{array}{l}
p \\
r
\end{array}\right) \cdot\left(\begin{array}{l}
q \\
s
\end{array}\right) \cdot(-\bar{x})^{r} \cdot(-\bar{y})^{s} \cdot m_{p-r, q-s} \quad \text { with } \quad \bar{x}=\frac{m_{10}}{m_{00}}, \bar{y}=\frac{m_{01}}{m_{00}}
$$

Scale and illumination intensity invariance is achieved by calculating a new set of moments $n_{p q}$ from the central moments $\mu_{p q}$ using

$$
n_{p q}=\frac{\mu_{p q}}{\mu_{00}} \cdot\left(\frac{\mu_{00}}{\mu_{20}+\mu_{02}}\right)^{\frac{p+q}{2}}
$$

Rotation invariance is slightly more difficult to achieve. The NCMC feature vector, which provides the desired rotation invariance properties, will be presented next.

\section{Normalized Complex Moment Components}

The NCMC is based on the theory of complex moments, first introduced by AbuMostafa and Psaltis in [14]. Abu-Mostafa and Psaltis demonstrate that only the magnitudes and not the phases of complex moments remain invariant under rotations, although both magnitudes and phases contain equally descriptive information regarding the image. The NCMC overcomes this by subjecting the complex moment phases to a suitable normalization procedure which makes them rotation invariant and, hence, utilizes all the available information regarding the image that is contained within the complex moments.

The first step in the calculation of the NCMC is to calculate the complex moments of the image. The complex moments $C_{p q}$ of an image $g(x, y)$ in terms of its geometric moments are investigated in [15] and are given by

$$
C_{p q}=\sum_{r=0}^{p}{ }_{s=0}^{q}\left(\begin{array}{l}
p \\
r
\end{array}\right) \cdot\left(\begin{array}{l}
q \\
s
\end{array}\right) \cdot i^{p+q-r-s} \cdot(-1)^{q-s} \cdot m_{r+s, p+q-r-s}
$$


If the normalized moments $n_{p q}$ of (3) are used in (4) instead of $m_{p q}$ then the complex moments $C_{p q}$ will be invariant to changes in translation, scale and illumination intensity.

To demonstrate the phase normalization procedure of the NCMC the complex moments $C_{p q}$ must be expressed in terms of magnitude and phase, i.e.

$$
C_{p q}=\left|C_{p q}\right| \cdot e^{i \Phi_{p q}}
$$

Abu-Mostafa and Psaltis demonstrate in [14] that if the image is rotated by an angle $\theta$ then the new set of complex moments is

$$
C_{p q}^{\prime}=C_{p q} \cdot e^{i(p-q) \theta}=\left|C_{p q}\right| \cdot e^{i\left(\Phi_{p q}+(p-q) \theta\right)}=\left|C_{p q}^{\prime}\right| \cdot e^{i \Phi_{p q}^{\prime}}
$$

and so

$$
\begin{gathered}
\left|C_{p q}^{\prime}\right|=\left|C_{p q}\right| \\
\Phi_{p q}^{\prime}=\Phi_{p q}+(p-q) \theta
\end{gathered}
$$

Relations (7) and (8) indicate that only the magnitudes, and not the phases, of complex moments are invariant with respect to rotation. In the NCMC, the complex moment phases are normalized and made invariant with respect to rotation by using relation (8).

For the calculation of the NCMC only the complex moments $C_{p q}$ with $p \geq q$ are considered, since $C_{q p}$ is the complex conjugate of $C_{p q}$ (i.e. $\Phi_{p q}=-\Phi_{q p}$ and $\left|C_{p q}\right|=\left|C_{q p}\right|$ ). After the magnitudes of these complex moments have been calculated, the complex polynomials $C_{p q}$ are divided into different groups. Each group $G_{k}$ contains only the complex moments $C_{p q}$ with $p-q=k$. For each group $G_{k}$ one complex moment $C_{r s}^{\text {ref }}(r-s$ $=k$ ) is selected to serve as a reference vector for that group. In this implementation, the reference vector for each group is the complex moment of the highest order $p+q$ within the group.

These reference vectors are used for the calculation of the relative phases $R \Phi_{p q}$, given by

$$
R \Phi_{p q}=\Phi_{r s}^{r e f}-\Phi_{p q} \quad(\text { with } p-q=r-s)
$$

When the image is rotated by an angle $\theta$ then, using relation (8), the new relative phases are given by

$$
\begin{aligned}
R \Phi_{p q}^{\prime} & =\Phi_{r s}^{r e f}-\Phi_{p q}^{\prime}=\Phi_{r s}^{r e f}+(r-s) \theta-\Phi_{p q}-(p-q) \theta= \\
& =\Phi_{r s}^{r e f}+(p-q) \theta-\Phi_{p q}-(p-q) \theta=\Phi_{r s}^{r e f}-\Phi_{p q}=R \Phi_{p q}
\end{aligned}
$$

This shows that the relative phases defined in (9) are invariant under object rotations. From (9) it becomes obvious that the reference vectors themselves have a zero relative phase. It should also be noted that the complex moments of group $G_{0}$ (i.e. $\left.C_{22}, C_{33}, \ldots\right)$ also have zero relative phases since by definition they have no imaginary 
component. The final step in the calculation of the NCMC is the transformation of the magnitudes and relative phases to Cartesian $(x, y)$ components which make up the final rotation invariant vector, using

$$
x=\left|C_{p q}\right| \cdot \cos \left(R \Phi_{p q}\right) \text { and } y=\left|C_{p q}\right| \cdot \sin \left(R \Phi_{p q}\right)
$$

\section{Hu Moment Invariants}

The results that will be presented later in this paper will perform a comparison between the NCMC vector and the commonly used Hu Moments Invariants (HMI). This section will briefly describe this rotation invariant feature vector.

Based on the theory of algebraic invariants, $\mathrm{Hu}$ was the first to introduce moment invariants ([10], [11]) for rotation invariant object recognition. The first step in the calculation of the HMI is the calculation of the complex polynomials $I_{p-r, r}$ in terms of the geometric moments of the image. The $I_{p-r, r}$ polynomials are given by

$$
I_{p-r, r}=\sum_{k=0}^{p-2 r}\left(\begin{array}{c}
p-2 r \\
k
\end{array}\right) \cdot(-i)^{k} \sum_{l=0}^{r}\left(\begin{array}{l}
r \\
l
\end{array}\right) \cdot m_{p-2 l-k, 2 l+k}
$$

with $p-2 r \geq 0$. The $\mathrm{Hu}$ rotational invariants are then calculated by combining these complex polynomials using

$$
I_{p r} \cdot I_{s q}^{t}+I_{r p} \cdot I_{q s}^{t}
$$

with $p-r=t(q-s)$. If the normalized moments $n_{p q}$ of (3) are used in (12) instead of $m_{p q}$ then the resultant rotation invariants will also be invariant to translation, scale and illumination intensity changes. Using moments up to the $3^{\text {rd }}$ order Hu derived a set of seven invariant features, historically known as the "Hu invariants". These are not included here but can be found in [11]. It is, however, possible to calculate $\mathrm{Hu}$ invariants using moments of higher orders, but their calculation becomes increasingly complex as the order increases. Wong et al. [16] describe an automatic approach for the calculation of higher order moment invariants, while Li [17] describes a method for deriving moment invariants of higher orders using the Fourier-Mellin transform.

\section{Results}

The data set used in this study can be seen in Fig. 1. This set splits into six classes, each class representing the same Printed Circuit Board with differences in particular components and their placement. The spatial resolution of these images is $256 \times 256$ pixels and their grayscale resolution is 256 levels. Note that all the classes have identical silhouettes, so the feature vectors will have to rely on differences in their grayscale texture information to successfully identify them. Each of these images was subjected to combinations of translation, rotation, uniform scale (between $-20 \%$ and $+20 \%$ ) and uniform illumination changes (between $-33 \%$ and $+33 \%$ ), thus creating 
400 images per class. For each class, 200 of those images were used for training and 200 for testing.
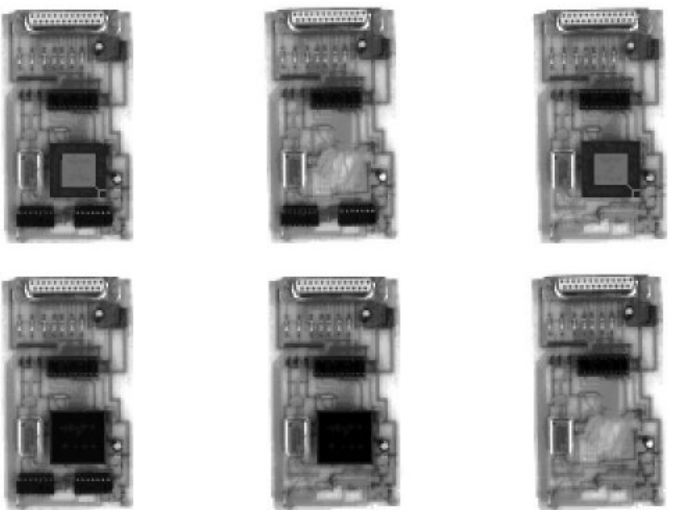

Fig. 1. Data set used for feature vector evaluation
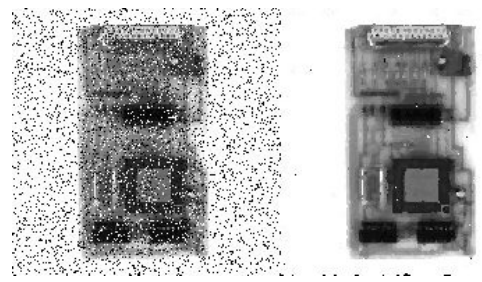

Fig. 2. An example of $20 \%$ noise addition (left) and subsequent median filtering (right)

The robustness of both the NCMC and HMI vectors to image distortions was also tested. Random 'salt \& pepper' noise was added onto the test images, which were subsequently filtered using a median filter, thus removing part of the noise but also blurring the images. The level of noise used was 20\%. Fig. 2 shows an example of $20 \%$ noise addition and subsequent filtering.

Geometric moments of the $3^{\text {rd }}$ up to the $6^{\text {th }}$ order were used in the implementation of both the NCMC and HMI vectors. Also, two different classification measures were used in conjunction with both feature vectors; a simple Euclidean distance classifier and a more complex weighted Euclidean distance classifier.

For the Euclidean distance classifier the distance measure is given by

$$
d_{i}=\sqrt{\sum_{j=1}^{n}\left(x_{j}-v_{i j}\right)^{2}}
$$


where $x$ is the test sample feature vector, $v_{i}$ is the $i^{\text {th }}$ class prototype feature vector (calculated during training) and $n$ is the number of features. The test sample is assigned to the class $i$ which minimizes $d_{i}$.

For the weighted Euclidean distance classifier the distance measure is given by

$$
d_{i}=\sqrt{\sum_{j=1}^{n} w_{j}\left(x_{j}-v_{i j}\right)^{2}}
$$

where $x, v$ and $n$ are the same as for the simple Euclidean classifier. The weighting factor $w_{j}$ is calculated for each feature $j$ and is the reciprocal of the mean of the $m$ standard deviations over the $m$ different classes for the $j^{\text {th }}$ feature, i.e.

$$
w_{j}=\frac{1}{\overline{s_{j}}} \text { with } \overline{s_{j}}=\frac{s_{1 j}+s_{2 j}+\ldots+s_{m j}}{m} \text { where } \mathrm{m}=\text { number of classes }
$$

This weighting factor has also been used by Cash and Hatamian in their OCR study [3]. The test sample is assigned to the class $i$ which minimizes $d_{i}$.

Tables 1, 2, 3 and 4 present comparative recognition results for the two feature vectors using different orders of moments and the two different classifiers. Tables 1 and 2 present the recognition results when no noise is added onto the images, while Tables 3 and 4 present the recognition results for the two feature vectors for the distorted images, i.e. after $20 \%$ noise addition and filtering.

Table 1. NCMC recognition results (in \%). Noise-free images

\begin{tabular}{ccccc}
\hline \multicolumn{5}{c}{ NCMC } \\
\hline & $6^{\text {th }}$ order & $5^{\text {th }}$ order & $4^{\text {th }}$ order & $3^{\text {rd }}$ order \\
\hline Euclidean & 100.0 & 100.0 & 100.0 & 98.5 \\
Weighted Euclidean & 100.0 & 100.0 & 100.0 & 98.6 \\
\hline
\end{tabular}

Table 2. HMI recognition results (in \%). Noise-free images

\begin{tabular}{ccccc}
\hline \multicolumn{5}{c}{ HMI } \\
\hline & $6^{\text {th }}$ order & $5^{\text {th }}$ order & $4^{\text {th }}$ order & $3^{\text {rd }}$ order \\
\hline Euclidean & 96.1 & 99.0 & 93.1 & 90.6 \\
Weighted Euclidean & 98.7 & 99.1 & 93.8 & 90.6 \\
\hline
\end{tabular}

It can be observed from Tables 1 and 2 that the NCMC delivers a higher recognition performance than the HMI. For the NCMC the best recognition scores are achieved using moments of the $4^{\text {th }}$ order or higher $\left(100 \%\right.$ recognition), while $3^{\text {rd }}$ order moments produce slightly lower results. The HMI performs worse than the NCMC and produces the best recognition scores using $5^{\text {th }}$ order moments $(99.1 \%$ recognition). 
It is also interesting to note that, for the NCMC vector, the simple Euclidean classifier delivers the same high performance as the more complicated weighted Euclidean classifier. For the HMI, the difference in performance between the two classifiers is not very big either, although the highest recognition scores are achieved with the more complex classifier only.

Table 3. NCMC recognition results (in \%). Distorted images

\begin{tabular}{ccccc}
\hline \multicolumn{6}{c}{ NCMC } \\
\hline & $6^{\text {th }}$ order & $5^{\text {th }}$ order & $4^{\text {th }}$ order & $3^{\text {rd }}$ order \\
\hline Euclidean & 99.0 & 99.3 & 98.8 & 94.8 \\
Weighted Euclidean & 99.3 & 100.0 & 98.7 & 94.2 \\
\hline
\end{tabular}

Table 4. HMI recognition results (in \%). Distorted images

\begin{tabular}{ccccc}
\hline \multicolumn{5}{c}{ HMI } \\
\hline & $6^{\text {th }}$ order & $5^{\text {th }}$ order & $4^{\text {th }}$ order & $3^{\text {rd }}$ order \\
\hline Euclidean & 72.9 & 95.8 & 83.3 & 77.5 \\
Weighted Euclidean & 87.7 & 96.0 & 86.2 & 77.5 \\
\hline
\end{tabular}

From Tables 3 and 4 it becomes clear that the proposed NCMC feature vector has a much higher tolerance to noise than the HMI feature vector. The NCMC is not greatly affected by the addition of noise, especially for moments of the $4^{\text {th }}$ order or higher, and still delivers very high recognition results (up to $100 \%$ recognition for $5^{\text {th }}$ order moments). In contrast, the HMI is more heavily affected by the addition of noise and produces much lower recognition results. For the HMI the highest recognition scores are produced by the $5^{\text {th }}$ order moments ( $96 \%$ recognition). It can also be noted that the NCMC delivers a very high recognition performance with both classifiers. For the HMI, however, the higher recognition scores are achieved only with the more complex classifier.

\section{Conclusions}

This paper has presented a new feature vector for statistical pattern recognition, the Normalized Complex Moment Components. The NCMC was evaluated and compared with the $\mathrm{Hu}$ moment invariants feature vector. The comparative results indicate that the NCMC has superior performance and greater tolerance to noise than the HMI vector. In addition to that, it was also found that the NCMC, used in conjunction with a computationally and conceptually simple classification measure such as the Euclidean distance, is capable of achieving performance levels that the 
HMI can only approach by employing a more complex classification measure, a fact which further illustrates the discriminating power of the proposed Normalized Complex Moment Components feature vector.

\section{References}

1. Loncaric, S.: A Survey of Shape Analysis Techniques. Pattern Recognition, Vol. 31, No. 8, pp. 983-1001, (1998)

2. Trier, O. D., Jain, A. K., Taxt, T.: Feature Extraction Methods for Character Recognition A Survey. Pattern Recognition, Vol. 29, No. 4, pp. 641-662, (1996)

3. Cash, G. L., Hatamian, M.: Optical Character Recognition by the Method of Moments. Computer Vision, Graphics and Image Processing, Vol. 39, pp. 291-310, (1987)

4. Balslev, I.: Noise Tolerance of Moment Invariants in Pattern Recognition. Pattern Recognition Letters, Vol. 19, pp. 1183-1189, (1998)

5. Teh, C. H., Chin, R. T.: On Digital Approximation of Moment Invariants. Computer Vision, Graphics and Image Processing, Vol. 33, pp. 318-326, (1986)

6. Shen, T. W., Lun, D. P. K., Siu, W. C.: On the Efficient Computation of 2-D Image Moments using the Discrete Radon Transform. Pattern Recognition, Vol. 31, No. 2, pp. 115120, (1998)

7. Yang, L., Albregsten, F.: Fast and Exact Computation of Cartesian Geometric Moments using Discrete Green's Theorem. Pattern Recognition, Vol. 29, No. 7, pp. 1061-1073, (1996)

8. Paschalakis, S., Lee, P.: The FPGA Design of a Moments Generator for Real Time Image Analysis Applications. In proceedings of the Fifth Canadian Workshop on FieldProgrammable Devices, 1998, pp. 83-87

9. Hatamian, M.: A Real-Time Two-Dimensional Moment Generating Algorithm and its Single Chip Implementation. IEEE Transactions on Acoustics, Speech and Signal Processing, Vol. ASSP-34, No. 3, pp. 546-553, (June 1986)

10.Hu, M. K.: Pattern Recognition by Moment Invariants. Proceedings of the IRE, September 1961, p. 1428

11.Hu, M. K.: Visual Pattern Recognition by Moment Invariants. IRE Transactions on Information Theory, February 1962, pp. 179-187

12.Dudani, S. A., Breeding, K. J.: Aircraft Identification by Moment Invariants. IEEE Transactions on Computers, Vol. C-26, No. 1, pp. 39-45, (January 1977)

13.Hsia, T. C.: A Note on Invariant Moments in Image Processing. IEEE Transactions on Systems, Man, and Cybernetics, Vol. SMC-11, No. 12, pp. 831-834, (December 1981)

14.Abu-Mostafa, Y. S., Psaltis, D.: Recognitive Aspects of Moments Invariants. IEEE Transactions on Pattern Analysis and Machine Intelligence, Vol. PAMI-6, No. 6, pp. 698706, (November 1984)

15.Teh, C. H., Chin, R. T.: On Image Analysis by the Method of Moments. IEEE Transactions on Pattern Analysis and Machine Intelligence, Vol. 10, No. 4, pp. 496-513, (July 1988)

16.Wong, W. H., Siu, W. C., Lam, K. M.: Generation of Moment Invariants and their uses for Character Recognition. Pattern Recognition Letters, Vol. 16, pp. 115-123, (February 1995)

17.Li, Y.: Reforming the Theory of Invariant Moments for Pattern Recognition. Pattern Recognition, Vol. 25, No. 7, pp. 723-730, (1992) 